\title{
Short-Range Ising Spin Glass: Multifractal Properties.
}

\author{
E. Nogueira Jr. \\ Departamento de Física, \\ Universidade Federal do Rio Grande do Norte, \\ CP 1641, 59072-970, Natal, RN, Brazil. \\ S. Coutinho \\ Laboratório de Fúsica Teórica e Computacional \\ Universidade Federal de Pernambuco, \\ 50670-901, Recife, PE, Brazil. \\ F. D. Nobre \\ Departamento de Física, \\ Universidade Federal do Rio Grande do Norte, \\ CP 1641, 59072-970, Natal, RN, Brazil. \\ E. M. F. Curadot \\ Centro Brasileiro de Pesquisas Físicas, \\ Rua Xavier Siguad 150, \\ 222290-180, Rio de Janeiro, RJ, Brazil. \\ J. R. L. de Almeida \\ Departamento de Física, \\ Universidade Federal de Pernambuco, \\ 50670-901, Recife, PE, Brazil. \\ (Accepted to Phys. Rev. E /01 March 97)
}

\begin{abstract}
The multifractal properties of the Edwards-Anderson order parameter of the short-range Ising spin glass model on $d=3$ diamond hierarchical lattices is studied via an exact recursion procedure. The profiles of the local order parameter are calculated and analysed within a range of temperatures close to the critical point with four symmetric distributions of the coupling constants (Gaussian, Bimodal, Uniform and Exponential). Unlike the pure case, the multifractal analysis of these profiles reveals that a large spectrum of the $\alpha$ Hölder exponent is required to describe the singularities of the measure defined by the normalized local order parameter, at and below the critical point. Minor changes in these spectra are observed for distinct initial distributions of coupling constants, suggesting an universal spectra behavior. For temperatures slightly above $T_{c}$, a dramatic change in the $F(\alpha)$ function is found, signalizing the transition.
\end{abstract}

05.50.+q, 75.10.Nr and 64.60.A

\section{INTRODUCTION}

The understanding of the nature of the spin-glass (SG) condensed phase in real systems has been challenging many authors [1], since the scenario emerging from Parisi's mean-field solution [2] of the SherringtonKirkpatrick (SK) [3] model came out. Some raised conclusions, like the structure of the free-energy barriers corresponding to many distinct phases below $T_{c}$ (pure states) [4, arranged in an ultrametric structure and the existence of a critical ordering field for the condensed phase [5], generated controversies that remain not satisfactorily elucidated. In particular, the domain-wall phenomenological scaling approach (droplet model) dismiss the SK model as appropriated for the description of short-range Ising spin glasses in low dimensions and does not share the same conclusions [6]7]. On the other hand, recent works based on numerical simulations presented results indicating that short-range models should exhibit the same qualitative features appearing in the SK model [8]. It is worth to mention that many efforts have been devoted to investigate exactly-solvable short-range SG models as an attempt to describe real spin glasses, where certain aspects of the system, e.g., the correlation length, the sensibility to the boundary conditions, and finite-size effects should present a very distinct behavior from those of infinite-range models. For the SG model on the pathological Bethe lattice with finite connectivity, the controversy about the nature of the condensed phase still persists. For instance, it was found that a replica-symmetric solution is stable for zero field when open (uncorrelated) boundary conditions are considered [9], while the breaking of replica symmetry is required to obtain a stable solution below $T_{c}$ when closed (correlated) boundary conditions are imposed to the system [10]. Another line of approach in the study of short-range SG behavior was developed after the work of Southern and 
Young [1] who succeeded to obtain, by using the MigdalKadanoff renormalization group (MKRG) scheme, phase diagrams showing the presence of a SG phase in three dimensions $(d=3)$, but not for $d=2$, indicating that the lower critical dimension $d_{l}$ should lie in this interval. This latter approach, which can be viewed as an approximation for real systems, was applied to investigate the exponents required to describe the transition to the condensed phase. On the other hand, based on the scaling theory, it was found that SG obeying symmetric distributions is characterized by four independent exponents, the thermal and the chaotic ones at $T=0$ and $T=T_{c}$ [12]. Within this approach the chaos exponents govern the sign-changing of the effective coupling of two spins, a distance $L$ apart, with the temperature. The interpretation for this phenomenon of critical chaos was done in the framework of the droplet theory [13].

Another point of view of the MKRG was explored since it was proved that its renormalization-group equations are exact for the Ising model on a family of diamond hierarchical lattices (DHL) [14]. The study of spin systems on such exotic lattices, whose coordination number varies from two to infinity, acquires relevance because exact solutions can be obtained and are well-controlled. Motivated by this, we generalized the method proposed by Morgado et al. [15] (MCC) to investigate the structure of the local Edwards-Anderson (EA) order parameter of the Ising SG model on these lattices. Through this method, we are able to calculate the local magnetization at each site by an exact recursion procedure and furthermore, the local EA order parameter associated to each site. The MCC method was firstly applied to the pure ferromagnetic Ising model on a two-connected DHL, revealing the multifractal structure of the local magnetization at the critical point [15]. Moreover, they showed that there is an infinite set of $\beta$ critical exponents for the local magnetization which are linearly related to the $\alpha$-Hölder exponent of the $F(\alpha)$ multifractal spectrum. This work was further generalized for a $p$-connected DHL with $q$ intermediated sites within each connection [16]. The MCC method was also applied to investigate the multifractal and critical properties of the q-state ferromagnetic Potts model on the $p$-connected DHL [17].

In the present paper we extend the preliminary results obtained by Coutinho et al., [18] where the $F(\alpha)$-spectra of the SG Ising model with an initial Gaussian distribution was studied for lattices up to twelve generations. Now we consider four distinct initial distributions of couplings (Bimodal, Gaussian, Uniform and Exponential), with lattices up to sixteen generations, investigating the temperature dependence of the $F(\alpha)$-spectrum around $T_{c}$. In section II, the model Hamiltonian is presented and the corresponding critical temperatures associated with each distribution are obtained. In section III, we give full details of the generalization of the MCC method for the SG case, as well as about the numerical procedure used to obtain the EA order parameter profiles for the four distinct initial distributions of couplings. In section
IV, the multifractal properties of the EA order parameter are obtained and the corresponding $F(\alpha)$-functions calculated by distinct initial distributions are compared. Furthermore, we investigate the temperature behavior of the boundaries of the $F(\alpha)$-functions, above and below the critical point, corresponding to each distribution. Finally, the conclusions are summarized in section V.

\section{THE HAMILTONIAN AND THE MIGDAL-KADANOFF RENORMALIZATION-GROUP PROCEDURE}

Let us consider the nearest-neighbor short-range Ising spin-glass model on a general $p$-connection diamond hierarchical lattice. The hierarchical lattices are connected graphs, recursively constructed by replacing all bonds at each generation by a basic unit, the starting point being the basic unit itself (first generation). The DHL basic unit is designed by two root-sites coupled through $p$ connections in parallel, each of them composed by two bonds in series via one internal site (scaling factor 2), as schematically shown in Fig. 1. The graph-fractal dimensions of such lattices are given by $d=1+\ln p / \ln 2$.

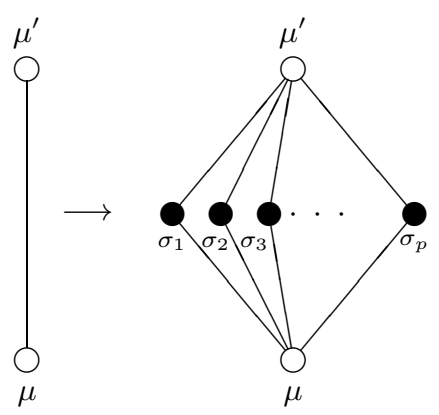

FIG. 1. General diamond hierarchical lattice basic unit, with $p$ parallel paths, each one composed by two bonds and one internal site (scaling factor 2).

The Hamiltonian of the present model is given by

$$
-\beta \mathcal{H}=\sum_{<i, j>} K_{N}(i, j) \sigma_{i} \sigma_{j}
$$

where $K_{N}(i, j)=-\beta J_{N}(i, j)$ is the reduced exchange coupling constant between nearest neighbor spins of a $N$-generation DHL, $J_{N}(i, j)$ being the $N^{t h}$-step MigdalKadanoff renormalized coupling constant obtained from the original distribution. It is worth to mention that the real-space Migdal-Kadanoff renormalization-group transformation on $d$-dimensional Bravais lattices is known to be equivalent to the exact solution on the DHL with a $d$ graph fractal dimension [14. The MKRG transformation of a $p$-connected DHL with general non-uniform coupling constants yields an effective coupling given by [11], 


$$
K_{N}^{e f f}\left(\mu, \mu^{\prime}\right)=\frac{1}{2} \sum_{l=1}^{p} \ln \left\{\frac{\cosh \left[K\left(\sigma_{l}, \mu\right)+K\left(\sigma_{l}, \mu^{\prime}\right)\right]}{\cosh \left[K\left(\sigma_{l}, \mu\right)-K\left(\sigma_{l}, \mu^{\prime}\right)\right]}\right\}
$$

which can be rewritten in terms of more convenient variables as

$$
t_{\mu, \mu^{\prime}}=\tanh \left[\sum_{l=1}^{p} \tanh ^{-1}\left(t_{\sigma, \mu} t_{\sigma, \mu^{\prime}}\right)\right],
$$

where $t_{x, y}=\tanh K_{x, y}$ is called thermal transmissivity. In Eq. (2) $\sigma_{l}$ labels the spins variables of the $p$ internal sites of a basic unit while $\mu$ and $\mu^{\prime}$ denote the root ones (see Fig. 1).

The critical temperature for the spin-glass model can be numerically obtained either from eqs.(2) or (3), by monitoring the width of the iterated distribution [11]. For the case of Eq. (3), in the zeroth step a pool of $M$ random initial transmissivities $\left(\left\{t_{i}\right\}=\tanh \left(\beta\left\{J_{i}\right\}\right)\right.$; $i=1,2, \ldots, M)$ is generated, with the coupling constants $\left\{J_{i}\right\}$ following a given probability distribution. In the first step Eq. (3) is iterated $M$ times by choosing at random $2 p$ initial transmissivities; the new $M$-valued pool represents the renormalized thermal transmissivity distribution. This process may be repeated and renormalized distributions can be numerically followed by computing its moments at each step [11] and a flow diagram may be constructed, e.g., in the transmissivity versus variance plane [19]. Since the spin glass fixed-point distribution is not analytically known, we considered four initial symmetric distributions of interest, namely the Gaussian, Bimodal, Exponential and Uniform ones, defined below,

$$
\begin{gathered}
P\left(J_{i, j}\right)=\frac{1}{\sqrt{2 \pi}} \exp \left(-\frac{1}{2} J_{i, j}^{2}\right) \quad(\text { Gaussian }), \\
P\left(J_{i, j}\right)=\frac{1}{2}\left[\delta\left(J_{i, j}-1\right)+\delta\left(J_{i, j}+1\right)\right] \quad \text { (Bimodal), } \\
P\left(J_{i, j}\right)=\frac{1}{\sqrt{2}} \exp \left(-\sqrt{2}\left|J_{i, j}\right|\right) \quad \text { (Exponential) }
\end{gathered}
$$

and

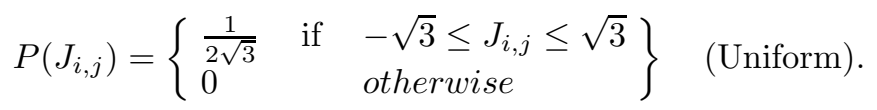

To each initial distribution a pseudo-critical temperature is associated, for which the flow will converge to the critical point characterized by the "fixed-point" distribution which is numerically known [21]. For temperatures very close but below (above) this pseudo-critical temperature the flow will at first, approach the "fixed point" and then turn on to the spin-glass (paramagnetic) fixed point characterized by infinite (zero) variance and zero mean. In Table 1, we present our numerical estimates for these pseudo-critical temperatures obtained from the initial distributions given by eqs.(位) in the case $d=3$ $(p=4)$, compared to other values reported in the literature.

\section{THE LOCAL MAGNETIZATION AND THE LOCAL EA ORDER PARAMETER}

\section{A. The generalized method for spin glasses}

The aim of this method is to establish a recursion relation between the value of the local magnetization of the internal site belonging to a connection of a given basic unit of the DHL with the local magnetization of its root sites. If this is achieved, one can consider a finite DHL with $N$ generations with Ising spin variables, described by the Hamiltonian given in Eq. (11), with nearestneighbor random exchange coupling constants chosen from a given initial distribution, and renormalize it $N-1$ times storing at each step all renormalized coupling constants. Then, taking arbitrarily initial magnetizations (corresponding to the spin-glass boundary conditions) for the root sites of the first generation, we can successively calculate the local magnetizations of each site of the $N$ generation DHL, at a given temperature and for a chosen initial coupling-constant distribution.

To obtain this recursion relation, let us consider a $N$ generation DHL and look at an arbitrary basic unit introduced at the $N^{t h}$-generation as shown in Fig. 2. This basic unit is connected to the lattice by its root sites $\left(\mu, \mu^{\prime}\right)$. Therefore, the partition function of the whole lattice can be written as

$$
\begin{aligned}
Z= & \operatorname{Tr}_{\left(\left\{\sigma_{i}\right\}, \mu, \mu^{\prime}\right)} \exp \left\{-\beta H^{\prime}\right\} \\
= & \operatorname{Tr}_{\left(\left\{\sigma_{i}\right\}, \mu, \mu^{\prime}\right)} \exp \left\{-\beta H\left(\left\{\sigma_{i}\right\}, \mu, \mu^{\prime}\right)\right\} \\
& \times \exp \left\{-\beta\left[h_{\mu} \mu+h_{\mu^{\prime}} \mu^{\prime}+K^{\prime} \mu \mu^{\prime}\right]\right\}
\end{aligned}
$$

where $\left\{\sigma_{i}\right\}, i=1,2 \ldots p$, denote the internal spins within each connection, $\mu, \mu^{\prime}$ are the root spins of the basic unit, $h_{\mu}, h_{\mu^{\prime}}$ and $K^{\prime}$ are, respectively, the effective fields and the effective coupling acting upon the basic unit root spins induced by the rest of the lattice. $H\left(\left\{\sigma_{i}\right\}, \mu, \mu^{\prime}\right)$ is the internal Hamiltonian of the basic unit given by

$$
H\left(\left\{\sigma_{i}\right\}, \mu, \mu^{\prime}\right)=\sum_{i=1}^{p}\left(K_{i} \mu+K_{i}^{\prime} \mu^{\prime}\right) \sigma_{i},
$$

where $K_{i}, K_{i}^{\prime}$ are the corresponding random coupling constants between the $\sigma_{i}$ 's and the $\mu, \mu^{\prime}$ spins, respectively. The local magnetizations of all sites within a given basic unit can be easily evaluated for the effective model

TABLE I. Spin-glass pseudo-critical temperatures for different probabilities distributions within the Migdal-Kadanoff renormalization-group procedure, in $d=3$ spin glass.

\begin{tabular}{|l|c|c|c|c|}
\hline \hline & Gaussian & Bimodal & Exponential & Uniform \\
\hline This work & 0.88 & 1.15 & 0.75 & 0.96 \\
Ref. [11 & $0.88 \pm 0.02$ & $1.05 \pm 0.02$ & - & $1.00 \pm 0.02$ \\
Ref. 119 & 0.85 & 1.2 & 0.7 & 1.0 \\
Ref. [12 & 0.89 & - & - & - \\
Ref. 20 & 0.83 & 1.15 & 0.71 & 0.94 \\
\hline \hline
\end{tabular}


defined by the Hamiltonian $H^{\prime}$ by tracing over all spins variables. However, since our main concern is to establish a recursion relation between the internal-site magnetization and these of the roots sites of a certain basic unit, the procedure can be further simplified by focusing our attention to a single connection and including the effects of the other connections onto the effective fields and coupling, following the ideas of the decoration transformation formalism [22]. In this case, our system is over-reduced to a single connection with an internal site under the action of the effective fields and effective coupling induced by the remaining lattice, as schematically shown in Fig. 2.

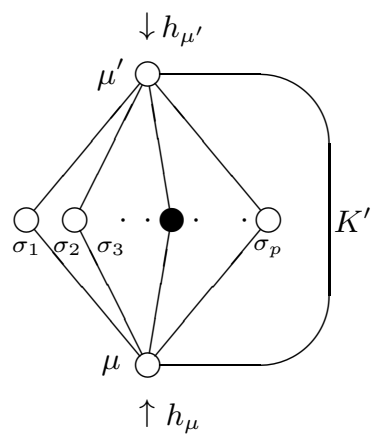

FIG. 2. Schematic representation of the equivalent system constructed by retaining a given basic unit of the final generation, with coupling constants $K_{i}(i=1, \ldots, 2 p) . \mu, \mu^{\prime}$ are the root spins of the basic unit, $h_{\mu}, h_{\mu^{\prime}}$ and $K^{\prime}$ are, respectively, the effective fields and the effective coupling generated by the whole lattice.

For this over-reduced system, the Hamiltonian in defined by

$H^{\prime \prime}\left(\sigma, \mu, \mu^{\prime}\right)=\left(K_{1} \mu+K_{2} \mu^{\prime}\right) \sigma+h_{\mu} \mu+h_{\mu^{\prime}} \mu^{\prime}+K^{\prime} \mu \mu^{\prime}$.

The magnetizations at each site are straightforwardly calculated, giving

$$
\begin{aligned}
<\mu> & =Z^{-1} \operatorname{Tr}\left[\mu \exp \left(-\beta H^{\prime \prime}\right)\right] \\
& =\frac{\left(\tau_{\mu}+t \tau_{\mu^{\prime}}\right)+t_{1} t_{2}\left(\tau_{\mu^{\prime}}+t \tau_{\mu}\right)}{1+t \tau_{\mu} \tau_{\mu^{\prime}}+t_{1} t_{2}\left(t+\tau_{\mu} \tau_{\mu^{\prime}}\right)} \\
<\mu^{\prime}> & =Z^{-1} \operatorname{Tr}\left[\mu^{\prime} \exp \left(-\beta H^{\prime \prime}\right)\right] \\
& =\frac{\left(\tau_{\mu^{\prime}}+t \tau_{\mu}\right)+t_{1} t_{2}\left(\tau_{\mu}+t \tau_{\mu^{\prime}}\right)}{1+t \tau_{\mu} \tau_{\mu^{\prime}}+t_{1} t_{2}\left(t+\tau_{\mu} \tau_{\mu^{\prime}}\right)} \\
<\sigma>= & Z^{-1} \operatorname{Tr}\left[\sigma \exp \left(-\beta H^{\prime \prime}\right)\right] \\
= & \frac{t_{1}\left(\tau_{\mu}+t \tau_{\mu^{\prime}}\right)+t_{2}\left(\tau_{\mu^{\prime}}+t \tau_{\mu}\right)}{1+t \tau_{\mu} \tau_{\mu^{\prime}}+t_{1} t_{2}\left(t+\tau_{\mu} \tau_{\mu^{\prime}}\right)}
\end{aligned}
$$

where $t=\tanh \left(K^{\prime}\right), t_{i}=\tanh \left(K_{i}\right)(i=1,2)$ and $\tau_{\alpha}=$ $\tanh \left(h_{\alpha}\right)$ for $\alpha=\mu, \mu^{\prime}$. Now, from Eqs. (8) and (9) we can write the unknown variables $\left(t, \tau_{\mu}\right.$ and $\left.\tau_{\mu^{\prime}}\right)$ as a function of $\langle\mu\rangle$ and $\left\langle\mu^{\prime}\right\rangle$ and $Z$, and substitute them in Eq. (10) to end up with the recursive equation

$$
<\sigma>=\frac{t_{1}\left(1-t_{2}^{2}\right)}{1-t_{1}^{2} t_{2}^{2}}<\mu>+\frac{t_{2}\left(1-t_{1}^{2}\right)}{1-t_{1}^{2} t_{2}^{2}}<\mu^{\prime}>\text {. }
$$

We emphasize that if the sites of a hierarchical lattice are properly addressed, Eq. (11) establishes a recursive equation between the local magnetization of the sites belonging to the last generation and the ones of previous generations. Moreover, its coefficients do not depend on the unknown fields and couplings, but only upon the coupling constants belonging to the chosen connection. This result is the main achievement of this method.

\section{B. Numerical Procedure}

To analyze the structure of the local EA order parameter of our model, we should calculate $\left\langle\sigma_{i}\right\rangle^{2}$ for all sites and average them over many samples, yielding to

$$
q_{i}^{E A}=\left[<\sigma_{i}>^{2}\right]_{c},
$$

where $[\ldots]_{c}$ stands for the configurational average taken over many independent initial distributions of couplings. To consider larger lattices we have to go further in the renormalization steps. Since the number of sites and bonds increases like $(2 p)^{N}$, the amount of computer memory required to store the magnetizations and the coupling constants during the intermediate steps will increase with such rate. In order to maximize the number of renormalization steps, we look at the magnetization structure of a subset of representative sites of the lattice. These sites are the $2^{N}$ ones belonging to any shortest path connecting the roots sites. The magnetization (and/or the EA local order parameter) structure of this subset can be viewed as a representative profile of the whole lattice. Since they are stochastically equivalent, we argue that by averaging over many profiles, we should obtain the correct scenario for the local EA order parameter of the considered model.

To calculate the profile of the local magnetization of a $N$ generation hierarchical lattice we make use of Eq. (11). To display the profile, we have to label the sites of a given path, assigning the values of its local magnetizations of the support set, due to the graph topological nature of the hierarchical lattices. To proceed, we choose the set of site labels by $(s, l)$ belonging to the interval $[0,1]$, defined by $s \times 2^{-l}, s=1,3,5, \ldots,\left(2^{l}-1\right)$, and $l$ labeling the generation $(l=1,2, \ldots, N)$. For this choice the recursive equation can be written as

$$
<\sigma>_{s, l}=\Lambda_{s_{1}, l_{1}}<\mu>_{s_{1}, l_{1}}+\Lambda_{s_{j}, l_{j}}<\mu^{\prime}>_{s_{j}, l_{j}}
$$


where $s_{1}=\frac{1}{2}(s \pm 1), l_{1}=l-1, s_{j}=\frac{1}{2}(s \mp 1), l_{j}=l-j$, with $j=2,3, \ldots, l$. The coefficients of Eq. (13) are given by

$$
\begin{aligned}
& \Lambda_{s_{1}, l_{1}}=\frac{t_{s, s_{1}}\left(1-t_{s, s_{j}}^{2}\right)}{1-t_{s, s_{1}}^{2} t_{s, s_{j}}^{2}} \\
& \Lambda_{s_{j}, l_{j}}=\frac{t_{s, s_{j}}\left(1-t_{s, s_{1}}^{2}\right)}{1-t_{s, s_{1}}^{2} t_{s, s_{j}}^{2}}
\end{aligned}
$$

where $t_{s, s_{j}}=\tanh \left[K_{l}\left(s, s_{j}\right)\right], K_{l}\left(s, s_{j}\right)$ being the coupling constant between the spins at the positions $s .2^{-l}$ and the one at $s_{j} .2^{-l_{j}}$.

To calculate an EA order parameter profile we must first generate the coupling constants for each level. Due to the disordered nature of the profile we make use of an equivalent stochastic procedure in order to save computer memory at intermediate steps of the calculation. For a fixed value of the temperature we create an initial distribution for the thermal transmissivities, represented by a pool of $M$ random numbers $\left(M \cong 10 \cdot 2^{N}\right)$. At the $N$ th level we choose randomly, from the initial distribution, a set of $2^{N}$ couplings, which are stored to be used later in the calculation of the site magnetizations. At the next level [ $N-1)$ th level], we obtain a renormalized distribution (new $M$ random numbers), generated according to the renormalization Eq. (3); from this distribution, we pick randomly $2^{N-1}$ couplings, which are also stored. This process is carried for $N-1$ times, such that at the last level, only two couplings are stored. Now, we make use of Eq. (13), fixing the initial values for the magnetization of the roots (zeroth generation) and calculate the local magnetization of each level, using for the coupling constants the values previously stored.

\section{The EA order parameter profiles}

For each of the initial distributions defined in Eq. (4), we generated profiles at the corresponding critical temperature $T_{c}$ (see table [), as well as $T_{1}=0.9 T_{c}$, $T_{2}=0.8 T_{c}$ and $T_{3}=0.7 T_{c}$. This was done for lattices with $d=3$ and $N=8$ up to 16 hierarchies. In Fig. 3 we display these profiles for the Gaussian distribution at the temperatures $T_{c}$ and $T_{1}(N=16)$ for just one sample, whereas in Fig. \#, the same is done for 200 samples. It is clear from these figures that the disordered structure of the local EA order parameter of one sample increases as we go further in the condensed phase. However, when the configurational average is taken, the profile presents uniformities reminiscent of the graph lattice symmetry, similar to what happens for the pure model [15]. It is also evident from Fig. 4 , the increasing of $q^{E A}=2^{-N} \sum_{i} q_{i}^{E A}$ , the mean value per site, as we decrease the temperature. For all other distributions listed above (Bimodal, Exponential and Uniform), the same qualitative behavior was observed [23].
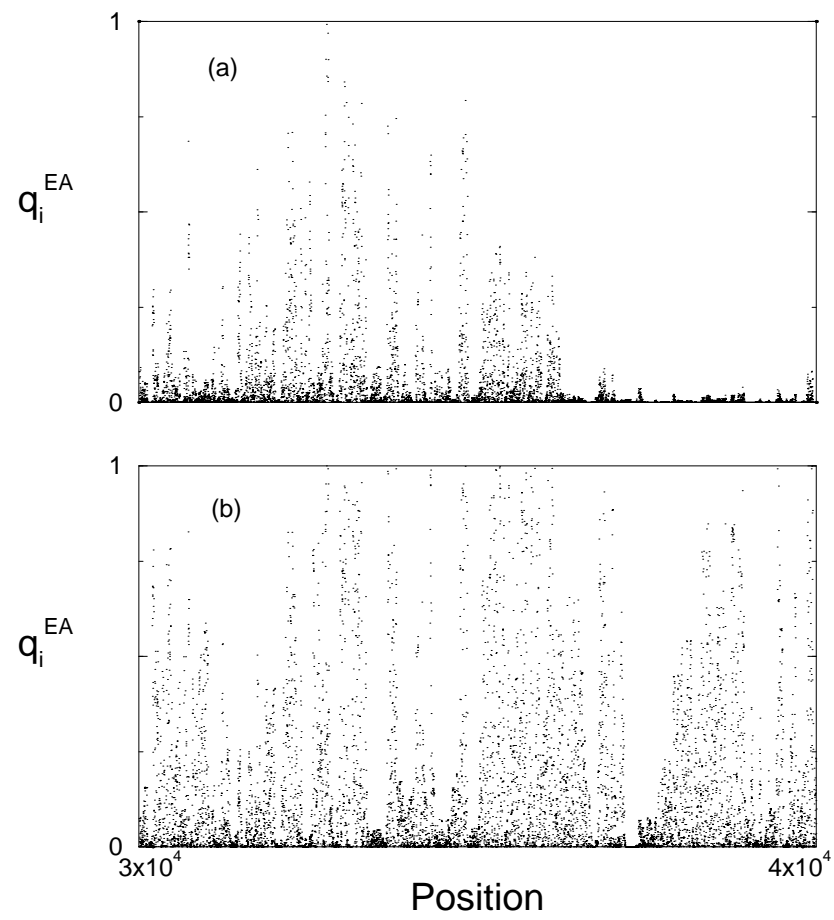

FIG. 3. Portion of the profile of local EA order parameter for one sample and a lattice with $N=16$ generations; the sites chosen belong to a sub-set with positions in the range $i=3.0 \times 10^{4}, \ldots, 4.0 \times 10^{4}$, selected from the set $i=1,2, \ldots, 2^{N}$, corresponding to a given shortest path connecting the two roots sites. (a) $T=T_{c}$. (b) $T=0.9 T_{c}$.

\section{THE MULTIFRACTAL PROPERTIES}

The high degree of discontinuity shown in the profiles suggests us the multifractal analysis as a tool to investigate the singularities of the measure constructed from the EA local order parameter, following the same approach used for pure models [15 17]. To obtain the multifractal spectra $(F(\alpha)$-function), we first define a measure by the normalized local EA order parameter

$$
\zeta_{i}^{E A}=\frac{q_{i}^{E A}}{\sum_{i} q_{i}^{E A}},
$$

and construct a parametrized family of normalized measures defined by

$$
\mu_{i}^{E A}=\frac{\left(\zeta_{i}^{E A}\right)^{q}}{\sum_{i}\left(\zeta_{i}^{E A}\right)^{q}} .
$$



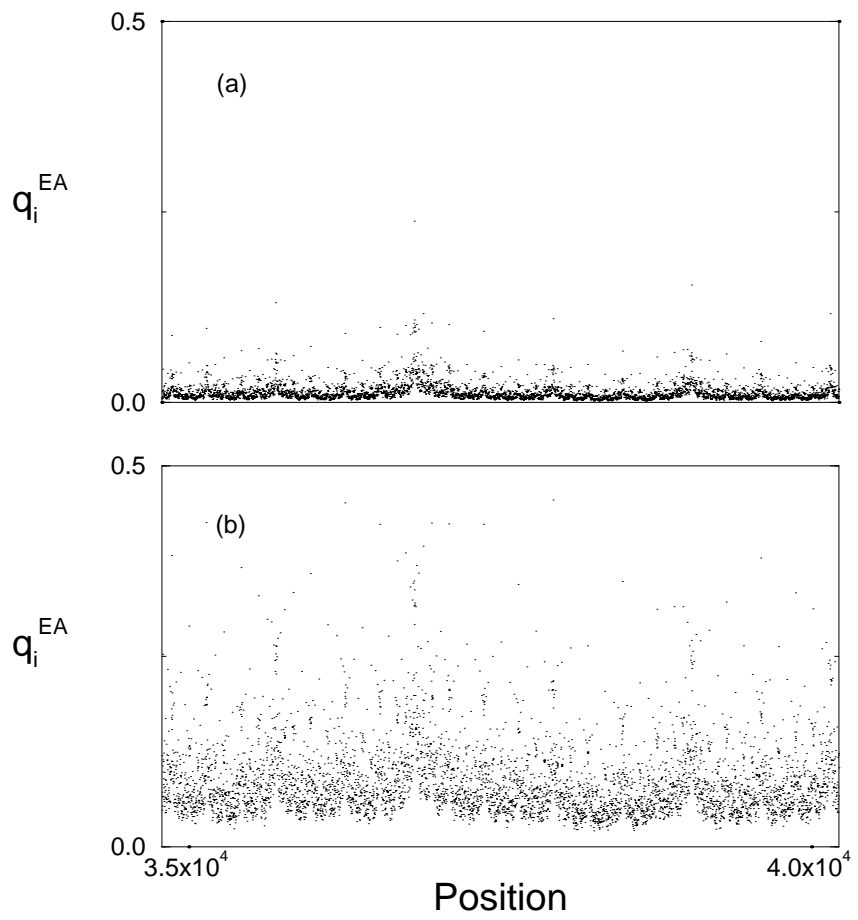

FIG. 4. Portion of the profile of the average local EA order parameter (200 samples) for a lattice with $N=16$ generations; the sites chosen are the same as in Fig. 3. (a) $T=T_{c}$. (b) $T=0.9 T_{c}$.

The $F(\alpha)$-function is now obtained following the method due to Chhabra and Jensen [24], where the spectrum is obtained by varying the parameter $q$ and calculating

$$
\begin{gathered}
F\left(\alpha_{q}\right)=\lim _{N \rightarrow \infty}\left\{\frac{-1}{N \ln 2} \sum_{i} \mu_{i}^{E A} \ln \mu_{i}^{E A}\right\}, \\
\alpha_{q}=\lim _{N \rightarrow \infty}\left\{\frac{-1}{N \ln 2} \sum_{i} \mu_{i}^{E A} \ln \zeta_{i}^{E A}\right\} .
\end{gathered}
$$

In Fig. 5, we display the corresponding $F(\alpha)$-functions for the profiles of one sample obtained from the initial distributions listed in Eq. (4), for temperatures at and below $T_{c}$. We notice light variations of the spectra by changing the temperature for the Gaussian, Bimodal and Uniform cases, whereas more pronounced changes are observed for the Exponential case. In Fig. 6, the corresponding $F(\alpha)$-functions averaged over 200 samples are shown for the Gaussian and Bimodal distributions at $T_{c}$ and $T_{1}=0.9 T_{c}$. Minor changes are observed when we compare with Fig. 5 .
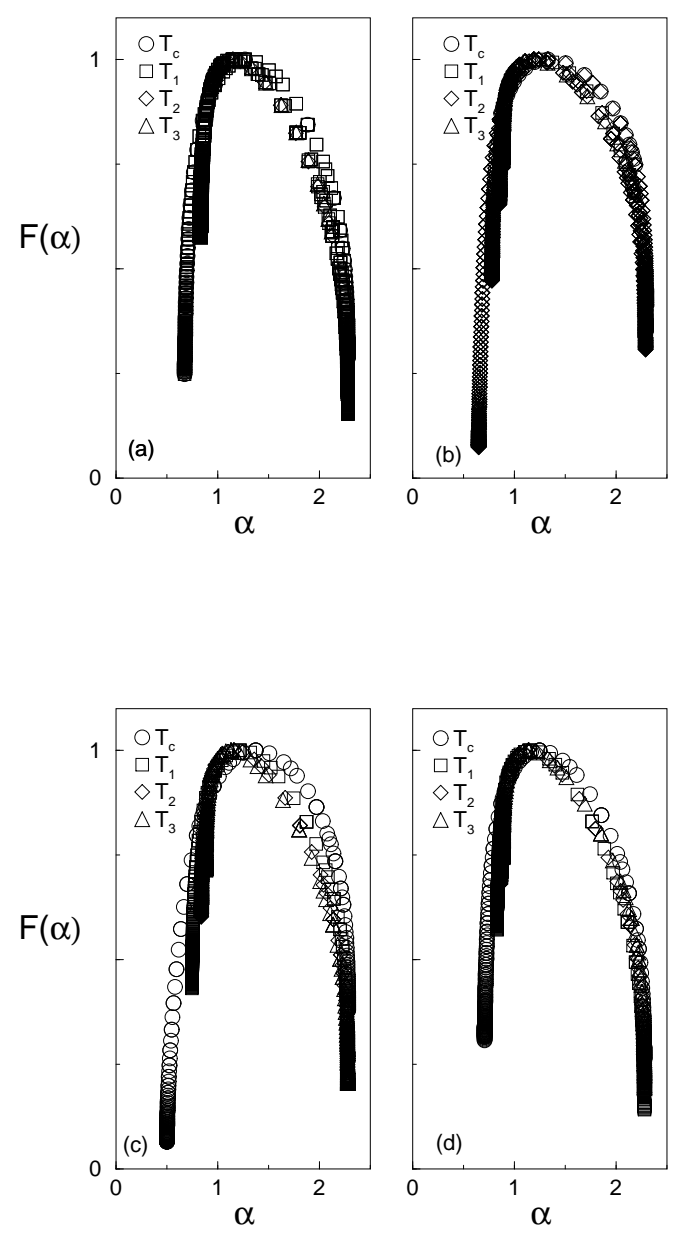

FIG. 5. $F(\alpha)$-functions of the local EA order parameter profile with one sample, for decreasing temperatures and different initial distributions of coupling constants. (a) Gaussian, (b) Bimodal, (c) Exponential and (d) Uniform distributions $\left(\bigcirc T=T_{c}, \square T_{1}=0.9 T_{c}, \diamond T_{2}=0.8 T_{c}\right.$ and $\triangle$ $\left.T_{3}=0.7 T_{c}\right)$.

In Fig. 7, we show in the same plot the $F(\alpha)$-functions at and below $T_{c}$ for the four considered distributions. It is worth to call the reader's attention to the universal character of the $F(\alpha)$-function within small deviations. Here we remark that although we have used the renormalized distributions of couplings at each step of the calculation, the influence of the initial distribution should be relevant, since in the thermodynamic limit $(N \rightarrow \infty)$, half of number of sites in the profile belong to generation $N$ and their magnetizations are calculated with the coupling constants introduced by the initial distribution. For the whole lattice this influence should be even more relevant; in this case $\frac{7}{8}$ of the total number of sites belong to generation $N$. 

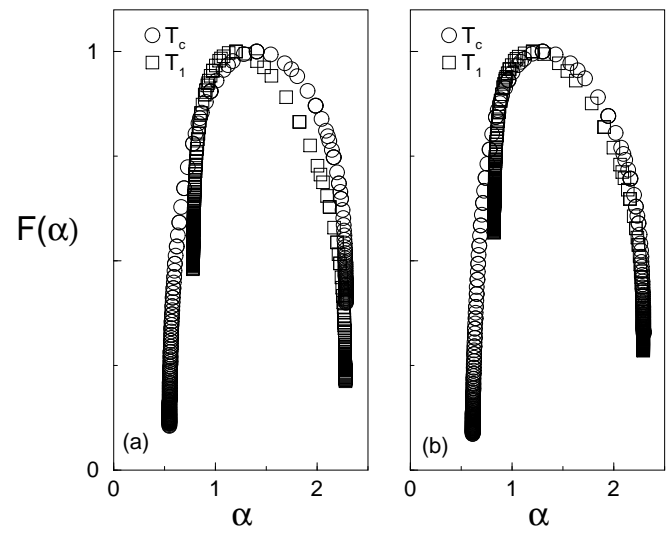

FIG. 6. $F(\alpha)$-functions of the average local EA order parameter profile (200 samples), at and below the critical temperature, for the (a) Gaussian and (b) Bimodal distributions of coupling constants $\left(\bigcirc T=T_{c}, \square T_{1}=0.9 T_{c}\right)$.
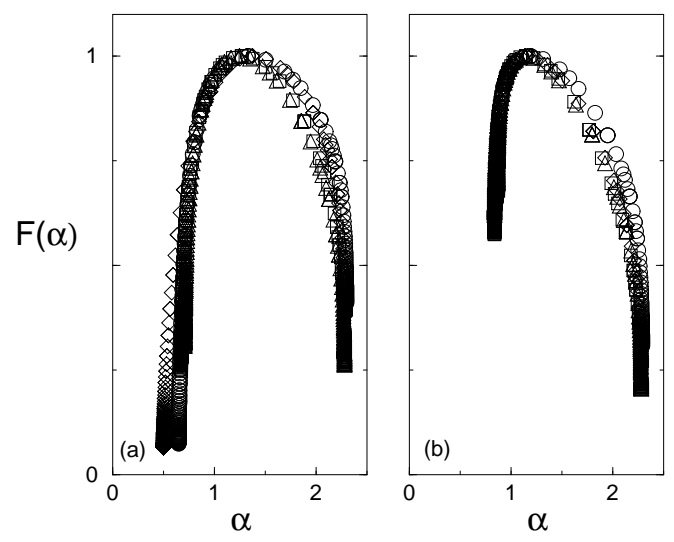

FIG. 7. Comparison between the $F(\alpha)$-functions of the local EA order parameter profiles at and below the critical temperature for the $\bigcirc$ Gaussian, $\square$ Bimodal, $\diamond$ Exponential and $\triangle$ Uniform initial distributions of coupling constants. (a) $T=T_{c}$ and (b) $T_{1}=0.9 T_{c}$.

In order to investigate the behavior of the $F(\alpha)$ spectrum around $T_{c}$, we plot in Fig. 8 the dependence of its upper and lower bounds as a function of the temperature, for all the considered initial distributions. We notice that below $T_{c}$ a constant value is observed for the upper bound $\left(\alpha_{\max }\right)$, while small deviations occur for the lower bound $\left(\alpha_{\min }\right)$ close to $T_{c}$. Nevertheless, for temperatures slightly above $T_{c}$ an abrupt increase is observed for the upper bound while a small decrease occurs for the lower bound, signalizing the $\mathrm{SG}$ transition. At $T \geq T_{c}$ the magnetization at the majority of sites vanishes, being eliminated from the calculation of the spectrum. Therefore, as we are dealing with a finite lattice one should expect a finite but higher $\alpha_{\max }$, which is the exponent governing the singularities of the set of smallest measures still present. This is evidenced by the rapid increasing of $\alpha_{\max }$ with the temperature, for $T>T_{c}$. On the other hand, the $\alpha_{\min }$ exponent which governs the set of higher measures should remain finite to describe the singularities of the measures belonging to the sites "close" to the root sites (or surface sites). Those sites are the ones whose magnetizations were calculated with at least one of the values imposed as initial boundary conditions. The intermediate points of the spectra should be spurious points, since the present algorithm [24] used to calculate the $F(\alpha)$ function is based on the method of moments and tend to produce a top envelop of the actual spectrum [25]. In the thermodynamic limit $(N \rightarrow \infty)$, we expect to have no spectrum except a point $(0,0)$ corresponding to the non-vanishing values introduced by the imposed boundary conditions.
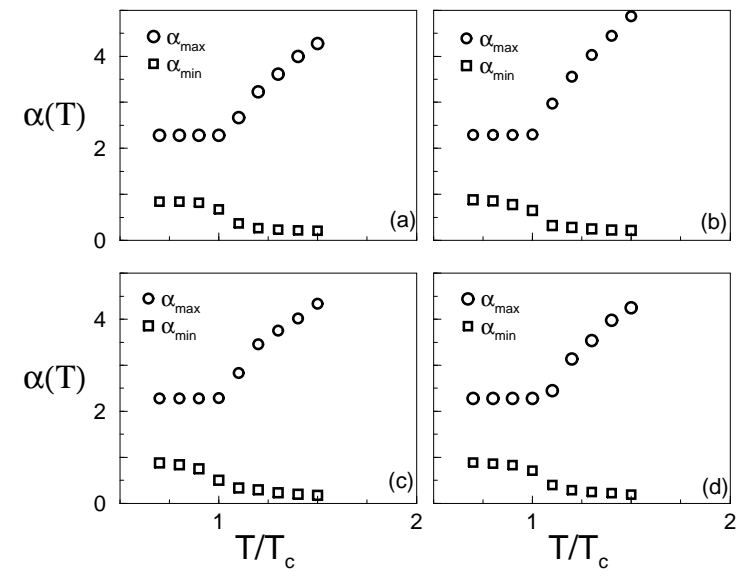

FIG. 8. Upper $\left(\bigcirc \alpha_{\max }\right)$ and lower $\left(\square \alpha_{\min }\right)$ bounds of the $F(\alpha)$ spectrum as a function of the temperature for different distributions of coupling constants. (a) Gaussian, (b) Bimodal, (c) Exponential and (d) Uniform distributions.

\section{CONCLUSIONS}

We generalized the exact recursion method developed by Morgado et al. [15] and applied it to investigate the structure of singularities of the local Edwards-Anderson order parameter of the short-range Ising spin-glass model on diamond hierarchical lattices. Within this procedure, the distribution of coupling constants is renormalized by the Migdal-Kadanoff renormalization equation, which is exact in such classes of lattices. The EA order parameter profiles $\left(2^{N}\right.$ sites) for lattices up to $N=16$ hierarchies were calculated by considering four types of initial distributions (Gaussian, Bimodal, Exponential and Uniform) and for temperatures around the critical point. For $T \leq T_{c}$ the profiles of the spin-glass condensed phase show a high degree of disorder and singularities increasing for lower temperatures. The multifractal analysis was applied to these profiles, revealing a large spectrum of exponents for the singularities of the measure defined by the normalized local EA order parameter. For each consid- 
ered distribution of couplings, the $F(\alpha)$-function of the profiles show slight variations with the temperature in the studied range of $0.7 T_{c} \sim T_{c}$, with larger deviations occurring when the temperature gets closer to $T_{c}$. These profiles reveal a high degree of local disorder of the spinglass condensed phase, a scenario not observed in the pure case [15]16] for the same classes of lattices. Moreover, small deviations are observed when the multifractal spectrum obtained from distinct initial distributions of coupling constants are compared at the same temperature. This suggests an universal multifractal behavior of the present model for distinct initial distributions of couplings, taking into account that $\frac{7}{8}$ of the total number of sites belong to the last generation, whose local magnetizations are calculated with the coupling constants introduced by the not-yet-renormalized (initial) distribution. We have also studied the temperature dependence of the range of the multifractal spectrum close to the critical point. For $T \leq T_{c}$ the range of the $\alpha$-Hölder exponent remains quite constant. However, when the temperature is higher than $T_{c}$, one observes an abrupt change in the multifractal spectrum signalizing the transition.

Contrary to the pure case, where a non-trivial multifractal behavior is observed only at the critical temperature [15, 16], the persistence of the $F(\alpha)$-function throughout the spin-glass phase indicates the highly nontrivial character of such phase. Although we are not able to associate the persistence of multifractality with any prediction from the available theories to describe shortrange spin glasses, the present work evidences the contrast between the spin-glass and ferromagnetic states and the critical nature of the spin-glass condensed phase.

\section{ACKNOWLEDGMENTS}

This research was supported by the CNPq, FINEP and CAPES (Brazilian governmental granting agencies). One of us (E. N. Jr.) is also grateful to FACEPE (Pernambuco state granting agency) for the financial support under the grant BFD-0505-1.05/95.

* Present address: International Centre for Condensed Matter Physics, Universidade de Brasília, CP 04513 , 70919-970, Brasília, DF, Brazil.

[1] For reviews, see for example K. Binder and A. P. Young, Rev. Mod. Phys. 58, 801 (1986); D. Chowdury and A. Moorkejee, Phys. Rep. 114, 1 (1984); A. J. Bray, Comments Condens. Mat. Phys. 14, 21 (1988); M. Mèzard, G. Parisi and M. A. Virasoro, Spin Glass Theory and Beyond, World Scientific, Singapore (1987); K. H. Fischer and J. A. Hertz, Spin Glasses, Cambridge Univ. Press, Cambridge (1991).
[2] G. Parisi, Phys. Rev. Lett. 43, 1754, (1979); 50, 1946 (1983); and M. Mèzard, G. Parisi, N. Sourlas, G. Thoulouse and M. Virasoro, J. Physique 45, 843 (1984).

[3] D. Sherrington and S. Kirkpatrick, Phys. Rev. Lett. 5, 965 (1975).

[4] Vertechi and M. Virasoro, J. Physique 50, 2325 (1989).

[5] J. R. L. de Almeida and D. J. Thouless, J. Phys. A 11, 983 (1978).

[6] A. J. Bray, J. Phys. C 15, L-57 (1982).

[7] D. S. Fisher and D. A. Huse, Phys. Rev. Lett. 56, 1601 (1986) and Phys. Rev. B 39, 373 (1988).

[8] J. D. Reger, R. N. Bhatt and A. P. Young, Phys. Rev. Lett. 64, 1859 (1990); A. Georges, M. Mèzard and J. S. Yedidia, Phys. Rev. Lett. 64, 2937 (1990); and E. R. Grannan and R. E. Hetzel, Phys. Rev. Lett. 67, 907 (1991); J. Wang and A. P. Young, J. Phys. A 26, 1063 (1993); E. Marinari, G. Parisi, F. Ritort and J. J. RuizLorenzo, Phys. Rev. Lett. 76, 843 (1996).

[9] D. J. Thouless, Phys. Rev. Lett. 56, 1082 (1986).

[10] P. Mottishaw, Europhys. Lett. 4, 33 (1987) and Lai PikYin and Y. Y. Goldshmidth, J. Phys. A 22, 399 (1989).

[11] B. W. Southern and A. P. Young, J. Phys. C 10, 2179 (1977).

[12] J. R. Banavar and A. J. Bray, Phys. Rev. B 35, 8888 (1987); H. J. Hilhorst and M. Nifle, Phys. Rev. Lett. 68, 2992 (1992) and Physica A 193, 48 (1993).

[13] M. J. Thill and H. J. Hilhorst, J. Phys. I (France) 6, 67 (1996).

[14] P. M. Bleher and E. Zalys, Commun. Math. Phys. 67, 17 (1979), and A. N. Berker and S. Ostlund, J. Phys. C 12, 4961 (1979).

[15] W. A. M. Morgado, S. Coutinho and E. M. F. Curado, J. Stat. Phys. 61, 913 (1990); Rev. Bras. Fis. (Brazil) 21, 247 (1991).

[16] O. Donato da Silva-Neto, MSc. Thesis, Universidade Federal de Pernambuco, (unpublished, 1992) and O. Donato da Silva-Neto and S. Coutinho, unpublished (1996).

[17] L. da Silva, E. M. F. Curado, S. Coutinho and W. A. M. Morgado, Phys. Rev. B 53, 6345 (1996).

[18] S. Coutinho, O. Donato-Neto, J. R. L. de Almeida, E. M. F. Curado and W. A. M. Morgado, Physica A 185, 271 (1992); and S. Coutinho, J. R. L. de Almeida and E. M. F. Curado, in Fractals in the Natural and Applied Sciences, M. M. Novak editor, North Holland, page 81 (1994).

[19] E. M. F. Curado and J-L. Meunier, Physica A 149, 164 (1988).

[20] L. Bernardi and I. A. Campbell, Phys. Rev. B, 49, 728 (1994).

[21] E. J. Hartford and S. R. McKay, J. Appl. Phys. 70, 6068 (1991).

[22] See, for example, M. E. Fisher, Phys. Rev. 113, 969 (1959).

[23] E. Nogueira-Jr. , PhD. Thesis, Universidade Federal de Pernambuco, (1996) unpublished.

[24] A. Chhabra and R. V. Jensen, Phys. Rev. Lett. 62, 1327 (1989).

[25] D. Veneziano, G. E. Moglen and R. F. Bras, Phys. Rev. E 52, 1387 (1995). 